Int. J. Electrochem. Sci., 14 (2019) 7037 - 7046

\title{
Simultaneous Determination of Dihydroxybenzene Isomers using Glass Carbon Electrode Modified with 3D CNT-graphene Decorated with Au Nanoparticles
}

\author{
Ti-Wei Chen ${ }^{l}$, Xiao-Na Yu ${ }^{l}$, Su-Juan $\mathrm{Li}^{2, *}$ \\ ${ }^{1}$ College of Chemistry and Chemical Engineering, Xuchang University, Xuchang 461000, Henan, \\ China \\ ${ }^{2}$ Henan Province Key Laboratory of New Optoelectronic Functional Materials, College of Chemistry \\ and Chemical Engineering, Anyang Normal University, Anyang, 455000, Henan, China \\ *E-mail: lemontree88@163.com
}

doi: $10.20964 / 2019.08 .58$

Received: 30 March 2019 / Accepted: 25 May 2019 / Published: 30 June 2019

3D CNT-graphene decorated with Au nanoparticles was modified onto glassy carbon electrode (Au NPs/CNTs/graphene/GCE) surface through casting and subsequent electrodeposition method. Morphological characterization of Au NPs/CNTs/graphene/GCE was performed by scanning electron microscopy (SEM), and evenly distributed Au NPs were observed to be anchored onto porous and 3D CNTs/graphene hybrids. The resultant electrode is used as an electrochemical interface for simultaneous determination of dihydroxybenzene isomers of hydroquinone (HQ), catechol (CC) and resorcinol (RC). Three well-resolved and sensitive peak signals were observed from cyclic voltammetric results attributing to the unique porous 3D nanostructure and high electrical conductivity of $\mathrm{Au}$ NPs/CNTs/graphene. Differential pulse voltammetry was used to perform quantitative detection of the three isomers. It was found that good performance of Au NPs/CNTs/graphene/GCE for detection of dihydroxybenzene isomers was obtained, including wide linear range, high sensitivity and selectivity, low detection limit and excellent stability. In addition, the proposed method can be successfully used for the determination of dihydroxybenzene isomers in real water samples.

Keywords: Dihydroxybenzene isomers; Carbon nanotube; Graphene; Gold nanoparticles

\section{FULL TEXT}

(C) 2019 The Authors. Published by ESG (www.electrochemsci.org). This article is an open access article distributed under the terms and conditions of the Creative Commons Attribution license (http://creativecommons.org/licenses/by/4.0/). 